\title{
Talla final en varones sintomáticos con hiperplasia suprarrenal no clásica tratados con glucocorticoides. Casos clínicos
}

\author{
Final height in symptomatic boys with late-onset adrenal hyperplasia (LOCAH), \\ treated with glucocorticoids. Clinical cases
}

\author{
Dra. Titania Pasqualini ${ }^{a}$, Dr. Guillermo Alonso ${ }^{a}$, Dra. Cecilia Fernández $z^{b}$ Lic. Noemí Buzzalino ${ }^{b}$ y \\ Dra. Liliana Dain ${ }^{b}$
}

\begin{abstract}
RESUMEN
La hiperplasia suprarrenal congénita no clásica puede ser asintomática o presentar signos de hiperandrogenismo y requerir tratamiento. Se describen las características clínicas en el diagnóstico, tratamiento y seguimiento hasta alcanzar la talla adulta en cuatro varones. La edad en el momento del diagnóstico fue de 9,2 a 11,6 años. Los motivos de consulta fueron pubarca precoz $(n=2)$, edad ósea acelerada $(n=1)$ y pubertad precoz $(\mathrm{n}=1)$. Todos los pacientes presentaron 17-hidroxiprogesterona elevada y el estudio molecular confirmó el diagnóstico. La edad ósea adelantada respecto de la edad cronológica $(13,1 \pm 0,5$ contra 10,2 $\pm 1,1 ; \mathrm{p}=0,008$ ) motivó el inicio del tratamiento con hidrocortisona. Durante el seguimiento, la media de talla disminuyó $1,4 \pm 0,4$ desviaciones estándar (DE) con respecto al diagnóstico $(p=0,007)$. Sin embargo, la media de talla final no difirió de la genética $(-0,9 \pm 0,7$ contra $-0,04 \pm 0,5 \mathrm{DE} ; \mathrm{p}=0,054)$. Conclusión: El tratamiento de los cuatro niños con adelanto de la edad ósea en el momento del diagnóstico permitió lograr una talla adulta que no difirió de la talla genética.

Palabras clave: hiperplasia suprarrenal congénita, tratamiento corticoideo, pubertad.
\end{abstract}

\section{SUMMARY}

Although corticoid replacement is recommended for thoselateonset adrenal hyperplasia with clinical manifestations, asymptomatic patients do not need treatment. We describe clinical features at diagnosis, treatment, and growth till adult- height, in 4 boys. At diagnosis, age ranged from 9.2-11.6 years. The initial symptoms/signs were: precocious pubarche $(n=2)$, accelerated bone age $(n=1)$ and precocious puberty $(n=1)$. All of them presented elevated 17 hydroxyprogesterone levels and were compound heterozygotes carrying p.V281L mutation. Since, at diagnosis, bone age was significantly advanced for chronological age $(13.1 \pm 0.5$ vs. $10.2 \pm 1.1 p=0.008)$, hydrocortisone therapy was initiated. During follow-up, mean height $\mathrm{Z}$ score decreased $1.4 \pm 0.4$ SDS ( $p=0.007)$, though adult mean height was not different from target height $(-0.39 \pm 0.7$ vs. $-0.04 \pm 0.5$ $\mathrm{SDS}, \mathrm{p}=0.054)$. In conclusion, in 4 symptomatic patients, accurate treatment of late-onset adrenal hyperplasia led to an adult

a. Sección Endocrinología, Crecimiento y Desarrollo. Departamento de Pediatría, Hospital Italiano.

b. CENAGEM, A.N.L.I.S e IBYME CONICET.

Correspondencia:

Dra. Titania Pasqualini: tpasqualin@intramed.net

Conflicto de intereses: Ninguno que declarar.

Recibido: 6-8-2012

Aceptado: 25-9-2012 mean height not different from target height. Advanced bone age at diagnosis and the loss of height during pubertal development suggest the need of therapy.

Key words: congenital adrenal hyperplasia, puberty, glucocorticoid treatment.

http:/ /dx.doi.org/10.5546/aap.2013.e35

\section{INTRODUCCIÓN}

La hiperplasia suprarrenal congénita no clásica (HSCNC) es una forma leve de HSC, con una prevalencia promedio de 1 en 1000 en los caucásicos. ${ }^{1,2}$ Los síntomas varían ampliamente; algunos pacientes pueden presentar pubarca precoz, acné, hirsutismo, irregularidades menstruales y alopecia, con infertilidad en la adultez o no. Muchos de ellos permanecen asintomáticos y no requieren tratamiento. ${ }^{2,3}$ El diagnóstico se basa en la detección de niveles elevados, tanto basales como posestimulación con corticotropina (ACTH), de 17-OH progesterona en la sangre. ${ }^{4}$

La enzima afectada, citocromo P450C21, es codificada por el gen CYP21A2 localizado en el cromosoma 6p21.3. Puesto que los pacientes con HSC poseen, en general, diferentes mutaciones en cada uno de sus alelos la expresión clínica de la patología dependerá del alelo menos afectado. ${ }^{1}$

Dadas las características de esta enfermedad, el diagnóstico de los varones con HSCNC es dificultoso y la sintomatología puede pasar inadvertida..$^{1,2,3,5}$ Algunos signos de alarma en la etapa prepuberal incluyen pubarca precoz y aceleración de la edad ósea., ${ }^{2,3}$ En la etapa puberal, el hallazgo de volumen testicular prepúber en presencia de edad ósea adelantada, con pubarca o sin ella, puede conducir al diagnóstico.

Se han informado pocos casos de varones con HSNC. El diagnóstico es dificultoso y puede pasar desapercibido. La falta de diagnóstico y, por lo tanto, la ausencia de tratamiento en estos pacientes plantea un riesgo en el inicio y la progresión adecuada del desarrollo puberal, con la 
consiguiente disminución del empuje de crecimiento en esta etapa, que puede conducir a un perjuicio sobre la talla adulta. ${ }^{2,3}$ Nuestro objetivo fue evaluar si estos cuatro pacientes con HSCNC, tratados con glucocorticoides, alcanzaban una estatura final cercana a la media de la talla objetivo genética.

\section{CASO CLÍNICO}

Se describen las características clínicas al diagnóstico, tratamiento y seguimiento hasta alcanzar la talla adulta en cuatro varones.

Se valoraron parámetros antropométricos, peso, puntaje de desviación estándar o puntaje $z$ (standard deviation score, SDS) de talla, SDS de talla genética, cambio de SDS de talla y la diferencia entre SDS de talla del paciente con respecto al SDS de talla genética. Todas las mediciones se compararon con las tablas nacionales de Lejarraga y Orfila. ${ }^{6}$

El desarrollo puberal se evaluó según los criterios de Marshall y Tanner.7

La edad ósea se evaluó por el método de Greulich y Pyle. ${ }^{8}$

Las mediciones de $17-\mathrm{OH}$ progesterona se realizaron por RIA, utilizando un kit comercial (DSL, Diagnostic System Laboratory, Houston, TX, EE.UU.).

Prueba de estimulación con ACTH: se realizó mediante la medición de cortisol y $17-\mathrm{OH}$ progesterona basales y a los 60 minutos luego del suministro intravenoso de $0,25 \mathrm{mg}$ de ACTH sintética (Synacthen Ò). Los valores superiores a 14 $\mathrm{ng} / \mathrm{ml}$ son sugestivos del diagnóstico hormonal de HSCNC. ${ }^{9,10}$

Estudio molecular de mutaciones en el gen CYP21A2: comprenden el estudio de las $10 \mathrm{mu}-$ taciones más frecuentes. ${ }^{9,10}$ En los pacientes en los que restaba determinar un alelo, se procedió a la secuenciación completa del gen. ${ }^{11}$ En presencia de la mutación p.Q318X, se realizó la técnica de Southern blot para descartar la presencia de un haplotipo con duplicación del gen. La segregación de los alelos afectados fue inferida a partir de los genotipos de los padres.

Pruebas estadísticas: todos los resultados se expresan en media \pm desviación estándar. La prueba de la t apareada se utilizó para comparar medias.

\section{RESULTADOS}

Los pacientes fueron diagnosticados entre los 9,2 y los 11,6 años por presentar pubarca $(n=2)$ (pacientes 1 y 4$)$, edad ósea acelerada $(n=1)$ (paciente 3$)$ y pubertad precoz $(n=1)$ (paciente 2$)$. Ninguno presentaba obesidad. Los cuatro pacientes mostraron un valor de $17-\mathrm{OH}$ progesterona basal o pos-ACTH elevado.

Todos los pacientes presentaron la mutación p.V281L en uno de sus alelos que predice una actividad enzimática (AE) entre $30 \%$ y $50 \%$. En el otro alelo, se hallaron mutaciones que predicen una $\mathrm{AE}$ entre $0 \%$ y $2 \%$ (paciente 1: p.R356W, paciente 2: p.IVS2-13A/C>G, paciente 4: p.Q318X), a excepción de un paciente con la mutación p.P482S, que predice una AE del 70\% (paciente 3).

En el momento del diagnóstico la media del SDS de talla fue mayor que el genético (media \pm desviación estándar 1,04 $\pm 0,8$ contra $-0,04 \pm 0,53$, diferencia $1,1 \pm 0,52 ; p=0,025$, prueba de la $t$ apareada).

La Tabla 1 muestra la edad cronológica y ósea en el momento del diagnóstico. La media de edad ósea se halló significativamente adelantada respecto de la cronológica: $13,1 \pm 0,5$ contra $10,2 \pm 1,1$; diferencia $2,9 \pm 0,9 ; p=0,008$. El SDS de talla para edad ósea al diagnóstico fue significativamente diferente del SDS de talla para edad cronológica $-1,04 \pm 0,62$ contra $1,04 \pm 0,8 ; p=0,006$; diferencia $2,08 \pm 0,58$.

TABLA 1. Características antropométricas y tiempo de tratamiento de los pacientes con hiperplasia suprarrenal congénita no clásica

\begin{tabular}{ccccccc}
\hline Paciente & $\begin{array}{c}\text { SDS de talla } \\
\text { al diagnóstico }\end{array}$ & $\begin{array}{c}\text { EC/EO (años) } \\
\text { al diagnóstico }\end{array}$ & $\begin{array}{c}\text { SDS de talla al } \\
\text { inicio puberal }\end{array}$ & $\begin{array}{c}\text { SDS de talla } \\
\text { al alcanzar la } \\
\text { talla adulta }\end{array}$ & $\begin{array}{c}\text { Tiempo de tratamiento } \\
\text { hasta alcanzar la talla } \\
\text { adulta (años) }\end{array}$ & $\begin{array}{c}\text { SDS de talla } \\
\text { genética }\end{array}$ \\
\hline 1 & 1,43 & $10,5 / 12,7$ & 0,93 & 0,29 & 5,32 y continúa tratamiento & 0,44 \\
2 & 0,65 & $9,4 / 13$ & 0,65 & $-1,18$ & 5,1 y continúa tratamiento & $-0,78$ \\
3 & 0,14 & $11,6 / 13,75$ & $-0,1$ & $-0,85$ & 4,2 y suspende tratamiento & $-0,26$ \\
4 & 1,95 & $9,2 / 13$ & 1,65 & 0,18 & 5,56 y suspende tratamiento & 0,34 \\
\end{tabular}

SDS: puntaje del desviación estándar; EC: edad cronológica; EO: edad ósea. 
En todos los pacientes el adelanto de la edad ósea al diagnóstico motivó el inicio del tratamiento con hidrocortisona. La dosis media utilizada fue de $10,7 \pm 2 \mathrm{mg} / \mathrm{m}^{2}$.

En los 4 pacientes la pubertad comenzó a los $11,1 \pm 1,5$ años con una edad ósea de 13,4 $\pm 0,4$ años $(p=0,0524)$. La ganancia de talla durante la pubertad fue de $23,6 \pm 4,5 \mathrm{~cm}$.

El paciente que ganó menos talla fue el que inició la pubertad con mayor edad ósea y es el que presentó un adelanto de ésta como único signo al diagnóstico (paciente 3).

La talla adulta se alcanzó luego de $5 \pm 0,6$ años de tratamiento (Tabla 1). La media de talla final no difirió de la genética $(-0,39 \pm 0,7$ contra $-0,04 \pm 0,5$ SDS; diferencia $-0,35 \pm 0,23 ; \mathrm{p}=0,054)$; sin embargo, la media de talla disminuyó $1,4 \pm 0,4$ SDS con respecto al diagnóstico $(p=0,007)$. La diferencia con la talla genética disminuyó de 1,1 $\pm 0,52$ SDS al diagnóstico a -0,35 $\pm 0,23$ SDS al alcanzar la talla adulta (Tabla 1).

Dos de los pacientes (pacientes 3 y 4) suspendieron el tratamiento con glucocorticoides después de finalizar el crecimiento.

\section{DISCUSIÓN}

Los niños con HSCNC producen cantidades normales de cortisol y aldosterona a expensas de una sobreproducción leve o moderada de precursores hormonales sexuales. Sin embargo, existe un amplio rango de gravedad en los síntomas clíni$\cos$, que varía entre formas asintomáticas ${ }^{5,12}$ hasta aquellas que se asocian con signos de hiperandrogenismo. ${ }^{1-3}$ Estos hallazgos sugieren que la sintomatología clínica es la que determina la necesidad de tratamiento.

Dos de los pacientes consultaron por pubarca precoz. Esta, que es el síntoma más frecuente que motiva la consulta en los niños con HSCNC, ${ }^{3,12}$ se define como la aparición del vello pubiano antes de los 8 años en las niñas y de los 9 años en los varones. En el $80 \%$ al $95 \%$ de los casos, es secundaria a la adrenarca temprana idiopática ${ }^{13}$ y en el $4 \%$ al $20 \%$ de los casos, es secundaria a HSCNC. ${ }^{14}$

A pesar de que nuestros pacientes presentaron talla normal o alta en la niñez, se describió que la talla final puede comprometerse por una fusión epifisaria temprana. ${ }^{3,12,15}$ Se observó que los varones con HSCNC tratados con glucocorticoides llegan a una talla final de $-0,8$ SDS, con una diferencia entre la talla genética y la talla final de $-0,35$ SDS. ${ }^{15}$ Nuestros pacientes sintomáticos con HSCNC, tratados con glucocorticoides, también alcanzaron una estatura final cercana a su talla objetivo genética.
Recientemente, Pijnenburg-Kleizen y cols. describieron que la edad ósea adelantada es uno de los signos más prominentes en los pacientes con HSCNC, secundario al aumento moderado de andrógenos, aún más notorio que la aceleración del crecimiento. ${ }^{12}$

Todos nuestros pacientes mostraron aumento de edad ósea al diagnóstico y en uno de ellos, fue el único signo inicial. La diferencia de SDS de talla para edad cronológica contra el SDS de talla para edad ósea mostró que el potencial de crecimiento, por efecto del adelanto de la maduración física, ya estaba comprometido. La asociación de edad ósea adelantada con síntomas de hiperandrogenismo o sin ellos, y con diagnóstico hormonal y molecular de HSCNC motivó el inicio del tratamiento con hidrocortisona en nuestros pacientes.

En resumen, la HSCNC es una enfermedad ampliamente subdiagnosticada, causante de una variedad de síntomas hiperandrogénicos que son factibles de tratar con terapia glucocorticoidea. En los niños con HSCNC, el adelanto importante de la edad ósea secundario al exceso de andrógenos conduce a una talla adulta baja y al aumento de riesgo de pubertad precoz. Esta última compromete adicionalmente la talla final. ${ }^{15}$

\section{CONCLUSIÓN}

En cuatro pacientes con HSCNC sintomáticos, el adelanto de la edad ósea motivó el inicio de tratamiento con glucocorticoides. Se logró alcanzar una estatura final cercana a la talla objetivo genética.

\section{BIBLIOGRAFÍA}

1. Merke DP, Bornstein SR. Congenital adrenal hyperplasia. Lancet 2005;365(9477):2125-36.

2. Speiser PW. Nonclassic adrenal hyperplasia. Rev Endocr Metab Disord 2009;10(1):77-82.

3. New MI. Extensive clinical experience. Nonclassical 21-Hydroxylase Deficiency. J Clin Endocrinol Metab 2006; 91(11):4205-14.

4. Azziz R, Dewailly D, Owerbach D. Clinical review 56: nonclassic adrenal hyperplasia: current concepts. J Clin Endocrinol Metab 1994; 78(4):810-5.

5. Nandagopal R, Sinaii N, Avila NA, Van Ryzin C, et al. Phenotypic profiling of parents with cryptic nonclassic congenital adrenal hyperplasia: findings in 145 unrelated families. Eur J Endocrinol 2011;164(6):977-84.

6. Lejarraga H, Orfila G. Estándares de peso y estatura para niños y niñas argentinos después del nacimiento hasta la madurez. Arch Arg Pediatr 1987;85:209-22.

7. Marshall WA, Tanner JM. Variations in the pattern of pubertal changes in boys. Arch Dis Child 1970;45(239):13-23.

8. Greulich WW, Pyle SI. Radiographic atlas of skeletal development of the hand and wrist. $2^{\text {nd }}$ ed Stanford CA: Stanford University Press; 1959.

9. Dain LB, Buzzalino ND, Oneto A, Belli S, et al. Classic and Nonclassical 21-Hydroxylase Deficiency: A Mole- 
cular Study of Argentine Patients. Clin Endocrinol (Oxf) 2002;56(2):239-45

10. Pasqualini T, Alonso G, Tomasini R, Galich AM, et al. Congenital Adrenal Hyperplasia: clinical characteristics and genotype in newborn, childhood and adolescense. Medicina (B Aires) 2007;67(3):253-61.

11. Minutolo C, Nadra AD, Fernandez C, Taboas M, et al. Structure-Based Analysis of Five Novel Disease-Causing Mutations in 21-Hydroxylase-Deficient Patients. PLoS One 2011;6(1): e15899.

12. Pijnenburg-Kleizen KJ, Borm GF, Otten BJ, Schott DA, et al. Absence of clinically relevant growth acceleration in untreated children with non-classical congenital adrenal hyperplasia. Horm Res Paediatr 2012;77(3):164-9.

13. Balducci R, Boscherini B, Mangiantini A, Morellini M, Toscano V. Isolated precocious pubarche: an approach. J Clin Endocrinol Metab 1994;79(2):582-9.

14. Armengaud JB, Charkaluk ML, Trivin C, Tardy V, et al. Precocious pubarche: distinguishing late-onset congenital adrenal hyperplasia from premature adrenarche. JClin Endocrinol Metab 2009;94(8):2835-40.

15. Balsamo A, Cicognani A, Baldazzi L, Barbaro M, et al. CYP21 genotype, adult height, and pubertal development in 55 patients treated for 21-hydroxylase deficiency. J Clin Endocrinol Metab 2003;88(12):5680-8. 